\title{
Inovação em subsidiárias de empresas multinacionais e a influência da matriz - um
} estudo de casos múltiplos

\author{
Mariane Figueira \\ Pós-doutora pelo Programa de Pós-Graduação em Administração da Universidade Federal de Lavras \\ mariane.figueira@gmail.com \\ Cristina Lelis Leal Calegario \\ Professora do Departamento de Administração e Economia da Universidade Federal de Lavras \\ ccalegario@dae.ufla.br \\ Paulo Henrique Moretti Luchesi \\ Analista de experiência do cliente na empresa MOIP. \\ phluchesi@gmail.com
}

\author{
Editor Científico: José Edson Lara \\ Organização Comitê Científico \\ Double Blind Review pelo SEER/OJS \\ Recebido em 11.09.2018 \\ Aprovado em 10.02.2019
}




\section{Resumo}

Título do trabalho: Inovação em subsidiárias de empresas multinacionais e a influência da matriz - um estudo de casos múltiplos

Objetivo do estudo: analisar como o relacionamento entre a matriz e a subsidiária de uma empresa multinacional e como a atuação da própria matriz podem influenciar no desenvolvimento de inovações na subsidiária;

Metodologia/abordagem: foi desenvolvido um estudo de casos múltiplos tendo como unidades-caso quatro empresas brasileiras de setores diferentes que foram adquiridas nos últimos dez anos por empresas multinacionais, se tornando subsidiárias;

Originalidade/relevância: o presente estudo contribui para preencher o gap encontrado na literatura relacionado, por um lado, aos desafios enfrentados pelas subsidiárias de multinacionais quanto à sua capacitação para inovar, e por outro lado, à necessidade de se conhecer mais sobre as ações e os mecanismos por meio dos quais a matriz pode desenvolver, promover e expandir as inovações na subsidiária;

Principais resultados: os resultados mostraram que os processos de aquisição que inseriram as empresas brasileiras nas redes das multinacionais se traduziram em benefícios para os dois lados, principalmente para as subsidiárias que após os processos de incorporação, tiveram algum tipo de inovação desencadeado.

Contribuições teóricas/metodológicas: As contribuições desse estudo estão relacionadas tanto ao quadro teórico elaborado que uniu as literaturas de negócios internacionais, transferência de conhecimento e gestão da inovação, quanto ao desenvolvimento de um estudo de casos múltiplos que analisou quatro casos distintos de empresas de setores industriais diferentes para analisar os fatores que influenciam a inovação na subsidiária.

Palavras-chave: negócios internacionais; inovação; subsidiárias de empresas multinacionais; relacionamento entre matriz e subsidiária.

\section{Abstract}

Title: Innovation in multinational subsidiaries and the parent company's influence - a multicase study

Objective of study: this study aimed at analyzing questions such as how the parent companysubsidiary relationship as well as the parent company's actions can influence the development of innovation within the multinational company's subsidiary;

Methodology: a multi-case study was developed, having as case units four Brazilian companies from different industrial sectors that were acquired in the past ten years by multinational companies, becoming subsidiaries;

Originality/relevance: this study contributes to fill the gap found in literature related, on the one hand, to the challenges faced by multinational subsidiaries regarding their ability to @ $@$ Revista Gestão \& Tecnologia, Pedro Leopoldo, v. 19, n. 5, p. 143-166, out./dez. 2019144 
innovate, and, on the other hand, to the need to understand more about the actions and mechanisms through which the parent company can develop, promote and expand innovations developed by the subsidiary;

Main results: results showed that the acquisition processes that incorporated the Brazilian companies in the networks of the multinationals resulted in benefits for both sides, especially for the subsidiaries that, after the merger processes, had different types of innovation triggered.

Theoretical/methodological contribution: the contribution of this study is related to both, the elaboration of a theoretical framework that united the literatures of international business, knowledge transfer and innovation management, as well as the development of a multi-case study that analyzed four distinct cases from different industry sectors to enable the analysis of the factors that influence innovation in the subsidiary.

Keywords: international business; innovation; subsidiaries of multinational companies; parent company subsidiary relationship.

\section{Resumen}

Título del artículo: Innovación en subsidiarias de empresas multinacionales y la influencia de la matriz - un estudio de casos múltiples

Objetivo del estudio: este estudio tuvo como objetivo analizar como la relación entre matriz y subsidiaria, y cómo la actuación de la propia matriz puede influir en el desarrollo de innovaciones en la subsidiaria.

Metodología: se desarrolló un estudio de casos múltiples, teniendo como unidades de caso cuatro empresas brasileñas de sectores diferentes que fueron adquiridas en los últimos diez años por empresas multinacionales, convirtiéndose em subsidiarias;

Originalidad/relevancia: este estudio contribuye a llenar el 'gap' que se encuentra en la literatura relacionado, por un lado, con los desafíos que enfrentan las subsidiarias con respecto a su capacidad para innovar y, por otro, con la necesidad de aprender más sobre acciones y mecanismos a través de los cuales la matriz puede desarrollar, promover y expandir innovaciones en la subsidiaria;

Resultados principales: Los resultados demostraron que los procesos de adquisición que introdujeron a las empresas brasileñas en las redes de las multinacionales se tradujeron en beneficios para ambas partes, principalmente para las subsidiarias que tras los procesos de incorporación tuvieron algún tipo de innovación desencadenada.

Contribuciones teóricas/metodológicas: las contribuciones de este estudio están relacionadas tanto con el marco teórico elaborado que unió las literaturas de negocios internacionales, transferencia de conocimiento y gestión de la innovación, como con el desarrollo de un estudio de casos múltiples que analizó cuatro casos distintos de compañías en diferentes sectores industriales para analizar los factores que influyen en la innovación en la subsidiaria. 
Palabras clave: negócios internacionales; innovación; subsidiarias de empresas multinacionales; relación entre matriz y subsidiaria.

\section{Introdução}

As inovações desenvolvidas nas subsidiárias de empresas multinacionais (EMNs) tem sido um tema mais explorado no presente, principalmente devido aos benefícios que podem ser repassados para a multinacional como um todo (Cantwell, 2016; Du \& Williams, 2017).

Alguns pesquisadores enfatizaram um novo cenário observado nos últimos dez anos em que o papel estratégico das subsidiárias é a produção de conhecimentos novos e inovação e a matriz tem atuado mais como receptora de conhecimento a partir de suas subsidiárias (Mudambi, Piscitello, \& Rabbiosi, 2014; Baglieri, Bruno, Vasconcellos, \& Grando, 2014; Najafi-Tavani, Zaefarian, Naudé, \& Giroud, 2015).

O potencial para inovar das subsidiárias pode estar condicionado ao tipo de recursos acessado por elas em duas fontes principais: contexto competitivo nacional e internacional (Costa, Borini, \& Amatucci, 2013), e transferência de conhecimento, knowhow de inovação e capacidades inovativas, da própria matriz (Almeida \& Phene, 2004).

Alguns estudos evidenciam que o relacionamento entre a matriz e suas subsidiárias é essencial para a criação de novos conhecimentos e tecnologias (Minbaeva, Peterson, Björkman, Fey, \& Park, 2003; Ciabuschi, Forsgren, \& Martín, 2017), todavia, pouca atenção tem sido dada às ações e aos mecanismos pelos quais a matriz pode desenvolver, promover e expandir as inovações na subsidiária.

Além disso, existem desafios significativos para que a capacitação para inovar possa ocorrer efetivamente. Estas barreiras surgem principalmente quando há diferenças entre crenças, pressupostos e normas culturais (Zander \& Kogut, 1995; Boh, Nguyen, \& Xu, 2013).

Desta forma, ainda há aspectos relacionados, tanto aos desafios enfrentados pelas subsidiárias quanto aos estímulos partindo da matriz, que carecem de maior investigação.

Nesse contexto, o principal objetivo do presente estudo foi analisar, por meio de um estudo de casos múltiplos, como o relacionamento entre matriz e subsidiária e como a atuação da própria matriz podem influenciar no desenvolvimento de inovação na subsidiária.

Esse estudo está divido em cinco partes. Após essa introdução, a seção dois apresenta o referencial teórico em que são expostos desafios enfrentados pela subsidiária para inovar, o relacionamento entre matriz e subsidiária e a atuação da matriz, como fatores que influenciam 
a geração de inovação. O referencial teórico também apresenta um quadro teórico para ilustrar esses fatores. Na seção três está apresentada a metodologia que guiou o presente e estudo. $\mathrm{Na}$ seção quatro são apresentados os resultados e discussão do estudo e na seção cinco, as considerações finais.

\section{Referencial teórico}

\subsection{Relacionamento entre matriz e subsidiária e geração de inovação na subsidiária}

Partindo das literaturas de negócios internacionais, transferência de conhecimento e gestão da inovação que colaboram para o entendimento da inovação em empresas multinacionais, o presente estudo também se propôs a elaborar um quadro teórico que procura ilustrar fatores que permeiam o relacionamento entre matriz e subsidiária e apontar a influência desses fatores na geração de inovação na subsidiária (Figura 1).

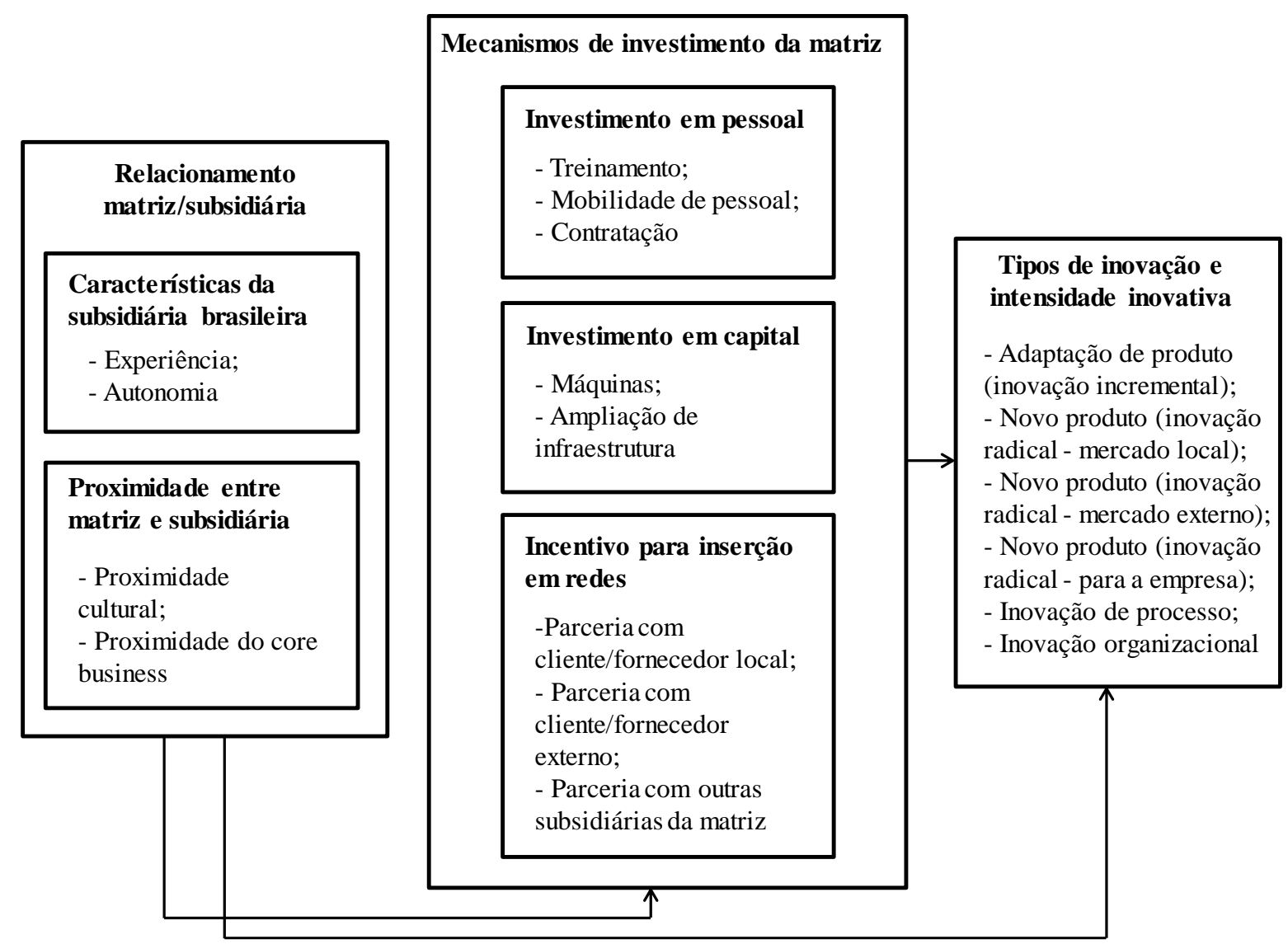

Figura 1 Fatores que influenciam a geração de inovação na subsidiária 
De acordo com Gavira (2008), nas últimas décadas, com o aumento da pressão por inovações, as EMNs passaram a descentralizar suas atividades, a fim de que cada uma de suas subsidiárias pudesse contribuir para a geração de conhecimento e inovação. Nesse novo cenário, as vantagens competitivas de que desfrutam as multinacionais já não partem apenas da matriz, mas também das inovações desenvolvidas pelas subsidiárias (Birkinshaw, Hood, \& Jonsson, 1998; Cantwell \& Mudambi, 2005).

Nesse contexto, o relacionamento da matriz de uma multinacional com suas subsidiárias é um fator decisivo para essas organizações, pois a capacidade de gerar e difundir conhecimento dentro da organização é uma fonte importante para a criação de valor ao qual empresas multinacionais podem ter acesso por meio de suas subsidiárias (Machado \& Bauer, 2014).

Outro fator importante para a geração de conhecimento novo e inovação na rede da multinacional é a transferência de conhecimento que pode ser considerada um processo mediado por alto nível de interação social. Por isso, torna-se fundamental a construção de laços sociais entre matriz e subsidiárias, além do desenvolvimento de um clima organizacional de confiança mútua (Silveira, Sbragia, Lopez-Vega, \& Tell, 2017).

De acordo com Schreiber, Vilela, Vargas e Maçada (2011), a eficiência da transferência de conhecimento é influenciada pela capacidade de absorção da subsidiária que pode ser definida como a habilidade de uma organização de reconhecer e assimilar o valor de uma nova informação externa e aplicá-la para fins comerciais (Cohen and Levinthal, 1990). A capacidade de absorver e combinar conhecimento é adquirida ao longo do tempo e com o aprendizado organizacional e alguns autores defendem que a subsidiária pode ser motivada a adquirir conhecimento que poderá romper com práticas organizacionais e rotinas de trabalho já estabelecidas para possibilitar a criação de conhecimento novo (Minbaeva, Peterson, Björkman, Fey, \& Park, 2003).

Ainda com respeito ao relacionamento entre matriz e subsidiárias, a forma como as EMNs administram, controlam, atribuem responsabilidades e dão autonomia às suas subsidiárias dispersas pelo mundo além de ser uma decisão estratégica, tem um impacto sobre as operações correntes e as perspectivas de crescimento das subsidiárias. Sendo assim, maiores graus de autonomia e descentralização para as subsidiárias lhes permite assumir um papel mais ativo na prospecção de novos conhecimentos e mercados e, consequentemente, na geração de inovação (Ferreira, Beltrão, \& Almeida, 2013). 
O conceito de autonomia segundo Boehe (2007) reflete a existência ou não da hierarquia organizacional na relação entre uma subsidiária e sua matriz. Conferir maior grau de autonomia às subsidiárias aumenta a receptividade local, contribuindo para a adaptação de produtos e processos ao contexto de negócios do país anfitrião. Nesse contexto, segundo a literatura, aquelas organizações que dispõem de maior autonomia têm maiores condições de se beneficiar de oportunidades de negócios e gerar inovações (Scott \& Gibbons, 2009; Kawai \& Strange, 2014).

A literatura que aborda as relações entre a matriz e as subsidiárias de empresas multinacionais e a geração de inovação nas subsidiárias também levanta temas como os desafios de se gerenciar a transferência de conhecimentos entre a matriz e subsidiárias localizadas em contextos geográficos e culturais distantes (Lucas, 2006; Qin, Ramburuth, \& Wang, 2008; Boh et al., 2013); e também os problemas de se administrar os relacionamentos entre a matriz e as subsidiárias se as subsidiárias estão localizadas em contextos econômicos diferentes (Beddi \& Mayrhofer, 2013). Sunaoshi, Kotabe, \& Murray (2005) explicaram que a transferência de tecnologia entre matriz e subsidiárias pode ser mais complexa quando as partes envolvidas no processo possuem diferenças culturais, que se manifestam principalmente no idioma.

Outros pesquisadores levantaram a importância do parentesco de negócios ou da proximidade da atividade principal desenvolvida pela matriz e pela subsidiária para a geração de inovação na subsidiária. Tang e Rowe (2012) observaram que o parentesco de negócios, definido como a medida com que uma subsidiária está relacionada com a atividade principal (core business) da sua matriz, tem um efeito positivo sobre o desempenho da subsidiária. Com uma amostra de 165 subsidiárias japonesas localizadas na China, no entanto, os pesquisadores observaram que as filiais modestamente relacionadas, superaram as subsidiárias independentes e as intimamente relacionadas, e que as filiais com uma relação mais próxima apresentaram desempenho fraco, especialmente, se a matriz tivesse uma participação maior na subsidiária e a subsidiária estivesse em sua fase inicial de operação no mercado hospedeiro.

$\mathrm{Na}$ literatura de gestão da inovação e negócios internacionais é possível também encontrar estudos colocando luz sobre a os impactos positivos das ações da matriz e sua contribuição para a geração de inovação na subsidiária (Ciabuschi, Dellestrand, \& Martín, 2011; Keupp, Palmié, \& Gassmann, 2011; Schleimer \& Pedersen, 2013). Segundo os autores 
mencionados, o relacionamento entre matriz e subsidiária pode contribuir positivamente para a inovação na subsidiária.

Além disso, existem formas por meio das quais a matriz de uma empresa multinacional pode estimular a transferência de conhecimento e a geração de conhecimentos novos e inovação na subsidiária (Piscitello \& Rabbiosi, 2006; Boehe, 2010).

Esses mecanismos podem ser divididos em três tipos: i) investimentos em pessoal (Piscitello \& Rabiosi, 2006) - evolvem mecanismos baseados em pessoas, como viagens e visitas, times, treinamentos, mobilidade de pessoal e contratações, favorecendo a transferência de conhecimento entre as pessoas; ii) investimentos em capital (Christensen, 1995; Cabral, 2007) - implementação de máquinas; ampliação de infraestrutura; criação de laboratórios tendo em vista que investimentos em ativos relevantes podem assegurar a produção de inovações tecnológicas, ou garantir a transformação de novas tecnologias em novos produtos e processos; e iii) incentivos para a participação de redes internas à empresa multinacional e redes externas (Costa, Borini e Amatucci, 2013) - a inserção em redes de relacionamentos se configura como uma oportunidade para as subsidiárias terem acesso a novos recursos e tecnologias. Esse debate com relação à importância da participação em redes é tratado pelos pesquisadores do chamado embeddedness ou enraizamento dual (Figueiredo, 2011; Ho, 2014; Achcaoucaou, Miravitles, \& León-Darder, 2016).

A questão do enraizamento dual tem recebido muita atenção nos últimos anos por tratar da inserção da subsidiária em redes de relacionamentos intra-corporativos, com a matriz e subsidiárias irmãs, bem como com parceiros externos que fazem parte do contexto local em que a subsidiária está instalada no país hospedeiro. Esses relacionamentos impactam as probabilidades da subsidiária de acessar conhecimento novo e, consequentemente, gerar diferentes tipos de inovação (Tsai, \& Wen, 2009; Figueiredo, 2011, Ho, 2014; Achcaoucaou, Miravitles, \& León-Darder, 2016).

O relacionamento entre a matriz e a subsidiária e os mecanismos para estimular a transferência e a geração de conhecimento podem colaborar para o desenvolvimento da capacidade inovativa da subsidiária e para a geração de diferentes tipos de inovação na subsidiária (Figueira, Luchesi, Silva, \& Calegario 2017). Entendendo que a inovação é a transformação e a utilização de novas ideias para fins de comercialização, ou simplesmente, dar utilidade social a novas ideias (Andreassi, 2007; Nemoto, Santos, \& Pinochet, 2018), a inovação é entendida nesse estudo na forma de produtos totalmente novos (inovação radical ou inovação 
disruptiva) para a empresa, para o mercado local ou para o mercado externo (OCDE, Eurostat, 2005; Andrade, Oliveira, Maccari, \& Hollnagel, 2018); na forma de produtos que receberam melhorias em sua fórmula ou em sua embalagem (inovação incremental) (Christensen, 1997), e também na forma de mudanças no modo de trabalhar e no local de trabalho, envolvendo novas tecnologias de informação e de comunicação, por exemplo, (inovação organizacional) (Moreira \& Queiroz, 2007).

A Figura 1 procurou ilustrar os fatores presentes no relacionamento entre matriz e subsidiária e as influências desses fatores na geração de diferentes tipos de inovação na subsidiária e também na intensidade inovativa (grau de novidade da inovação). O primeiro bloco da esquerda representa as características do relacionamento entre matriz e subsidiária, em que as características da subsidiária, como experiência e autonomia, e as características da proximidade entre matriz e subsidiária (proximidade cultural e proximidade do core business) impactam o bloco central - os mecanismos utilizados pela matriz para motivar a geração de inovação na subsidiária - e também a própria inovação na subsidiária. Esses mecanismos de investimento utilizados pela matriz (mecanismos de investimento em pessoal; mecanismos de investimento em capital; e incentivos para a participação da subsidiária em redes) afetam diretamente a inovação e a intensidade inovativa da subsidiária.

\section{Metodologia de pesquisa}

\subsection{Tipo de pesquisa}

Esta é uma pesquisa qualitativa (Strauss \& Corbin, 2008; Silva, Gaspar, Costa, \& Magalhães, 2018) em que foi desenvolvido um estudo de casos múltiplos (Yin, 2010) de quatro empresas inovadoras que foram fundadas em Minas Gerais e adquiridas nos últimos dez anos por empresas multinacionais estrangeiras, provenientes da Alemanha e da França, se tornando subsidiárias das redes dessas multinacionais.

O estudo de casos múltiplos é um método em que duas ou mais unidades-caso são utilizadas para investigar empiricamente um fenômeno contemporâneo em profundidade e em seu contexto de vida real (Yin, 2010). De acordo com Yin (2010), os estudos de caso podem 
cobrir casos múltiplos e, então, tirar um conjunto único de conclusões, como uma espécie de estudo de casos cruzados (“cross- case”).

O estudo de casos múltiplos foi desenvolvido a partir de dados coletados de fontes bibliográficas e documentais e 4 entrevistas em profundidade guiadas por roteiro semiestruturado e realizadas com gestores e diretores proprietários das subsidiárias pesquisadas nos meses de maio, junho e julho de 2017.

As empresas Unidades-caso dessa pesquisa são provenientes de setores diferentes e receberam os seguintes nomes fictícios: A, B, C e D. As empresas multinacionais que adquiriram as brasileiras pesquisadas também receberam nomes fictícios: A1, B1, C1 e D1.

\subsection{Análise e interpretação dos dados}

Para o desenvolvimento desse estudo as entrevistas realizadas, com duração de aproximadamente 60 minutos, foram gravadas, transcritas e analisadas com a aplicação da técnica de análise de conteúdo temática (Minayo, 2000), identificando citações dos entrevistados que deram suporte a categorias que foram desenvolvidas para analisar e interpretar os dados coletados e fazer as ligações do que foi encontrado na realidade com o quadro teórico (Figura 1) que foi criado a partir do que já foi construído na literatura (Corley \& Gioia, 2004).

As categorias de análise desenvolvidas a partir da leitura dos dados foram as seguintes: 1- trajetória histórica das empresas (unidades-caso); 2- proximidade do core business (atividade central) e impactos da aquisição para as empresas - matriz/adquirente e subsidiária/adquirida; 3- proximidade cultural; 4- autonomia diante da matriz; 5- motivação e incentivos recebidos da matriz para a geração de inovação.

\section{Resultados e discussão}

Em um primeiro momento serão apresentadas as trajetórias históricas de cada uma das empresas pesquisadas (A, B, C e D), seguidas de informações sobre as empresas multinacionais que adquiriram essas empresas, incorporando-as à rede dessas multinacionais. Então, serão abordados os temas que foram identificados dentro dos dados coletados nas entrevistas e que permitiram entender as relações entre a matriz e a subsidiária, algumas das mudanças ocorridas 
e o desenvolvimento das inovações que se seguiu. Nesse sentido, em primeiro lugar será apresentada a percepção sobre as proximidades entre as subsidiárias e matrizes pesquisadas, para em um segundo momento tratar da autonomia para atuar das subsidiárias e, por fim, das motivações e incentivos recebidos da matriz para inovar.

\subsection{Trajetória histórica das empresas (unidades-caso) dessa pesquisa}

\section{A Empresa A}

A empresa A, fundada em 2009, é uma evolução de uma ideia de negócio que surgiu em 2005, em Santa Rita do Sapucaí, Minas Gerais, com o então estudante de engenharia, fundador e atual CEO da A, de criar uma empresa de software e equipamentos de segurança eletrônica especializada no desenvolvimento de produtos para identificação e rastreamento de recémnascidos em maternidades. A empresa A é uma fabricante de produtos de segurança eletrônica com desenvolvimento em Santa Rita do Sapucaí e fabricação no polo industrial de Manaus, contando com mais de 200 funcionários. Em 2009, a A fez um acordo com a Sony Corporation para utilizar seus sensores de imagem em sua linha de câmeras de segurança. A partir desse ponto, a empresa também passou a fabricar câmeras e de 2009 para 2013 seu faturamento passou de 3 milhões para 47 milhões de reais.

No ano de 2013, a A teve $51 \%$ de suas ações compradas pela multinacional A1, multinacional francesa, presente atualmente em mais de 60 países e líder mundial em automação de portas e janelas. A aquisição da A pela A1 trouxe maior infraestrutura de gestão e tecnologia, além de abrir o mercado de exportação para a A. Em 2016, os primeiros donos da empresa A reinvestiram e reassumiram integralmente o controle da empresa e em 2017 uma empresa privada brasileira do segmento de eletrônicos e informática, anunciou a aquisição da A.

\section{A Empresa B}

No ano de 2008, em Belo Horizonte, Minas Gerais, três mineiros tiveram a ideia de criar uma solução que facilitasse o recebimento de pagamentos na internet e impactasse de maneira 
positiva o negócio de milhares de empreendedores digitais, criando a empresa B. No ano seguinte, a empresa recebeu investimentos dos acionistas Arpex, Ideiasnet e IG e foi transferida para São Paulo.

Em 2010, a empresa foi a primeira da América Latina a abrir APIs (Application Programming Interfaces) - uma API é um conjunto de estruturas de programação para acesso a um determinado sistema no mercado de pagamentos - uma iniciativa que tornou possível a criação de diversos modelos de negócios e contribuiu para o amadurecimento do ecossistema brasileiro de inovação. Dois anos depois, a B transformou o mercado de pagamentos ao lançar o conceito de 'Checkout Transparente', uma funcionalidade que turbinou a conversão de milhares de lojas virtuais.

No ano de 2016, a empresa B foi adquirida pela empresa multinacional alemã B1, uma empresa especialista em software e tecnologias de informação para terceirização e soluções de rótulos brancos para processamento de pagamentos e emissão de produtos. Fundada em 1999, com 16 anos de experiência no mercado, a multinacional alemã B1 fornece às empresas uma infra-estrutura que inclui licenças de emissão necessárias para produtos de cartão e conta. A multinacional B1 conta com mais de 25 mil clientes de várias indústrias, 3.600 funcionários, mais de 100 moedas de transação e conexão a mais de 200 redes internacionais de pagamentos (bancos, soluções de pagamentos, rede de cartões).

\section{A Empresa C}

A empresa C foi fundada no ano de 1995, na cidade de Uberlândia, Minas Gerais, para responder à necessidade de grandes grupos empresariais de administrar e firmar convênios que beneficiassem seus colaboradores. A terceirização desta administração resultou na otimização de custos para as empresas contratantes e beneficiou milhares de funcionários com uma ampla rede fornecedora de produtos e serviços. As operações da $\mathrm{C}$ eram feitas totalmente online e em real time para estabelecer de forma rápida e prática convênios entre empresas e estabelecimentos da cidade, tais como farmácias, supermercados e mercearias.

A empresa C foi se expandindo e em 2011, além de oferecer benefícios para colaboradores com os cartões convênio, alimentação, refeição, premiação e cartão presente, decidiu expandir suas operações para o setor de logística, criando um cartão destinado ao pagamento de frente eletrônico, um cartão combustível e o sistema de gestão de frotas. Para 
completar o portfólio de serviços voltados ao setor, no mesmo período, a companhia adquiriu uma plataforma especializada no rastreamento e telemetria de veículos.

Tendo sido considerada a quarta maior empresa do Brasil no segmento de benefícios em 2014 e construído grandes parcerias no país em 2015, chamou a atenção do Grupo C1, que no ano de 2016 adquiriu a empresa.

O Grupo C1, multinacional francesa, fundada no ano de 1964, é hoje o terceiro maior player do mercado global de benefícios, comercializando vales e serviços (cheques, cartões, aplicativos móveis, web, etc.) para facilitar o acesso à alimentação, cultura, lazer, educação, assistência a domicílio, ajuda social e também para acompanhar as empresas no gerenciamento de suas despesas profissionais ou no desenvolvimento de programas de fidelização.

\section{A Empresa D}

Fundada em 1975, a Empresa D, terceira maior empresa de ração para animais no mercado brasileiro, exporta para mais de 40 países dos cinco continentes e possui uma fábrica com capacidade de produção de 350.000 toneladas por ano. Localizada em Três Corações, Minas Gerais, a D conta com cerca de 1.400 funcionários e dispõe de uma rede comercial com 21 centros de distribuição. A empresa tem duas atividades principais: o petfood e os alimentos para animais de produção. Além disso, a empresa exporta para mais de 40 países e possui um centro de pesquisa mundialmente reconhecido em alimentos para animais de estimação em parceria com uma grande universidade americana no campo veterinário, a Universidade de Illinois, e se beneficia de um portfólio significativo de patentes.

No ano de 2014, a empresa foi adquirida pela multinacional D1, principal atuante francesa em nutrição e saúde animal. Esta operação confirmou a estratégia de desenvolvimento da D1 neste setor e permitiu que a multinacional obtivesse uma posição-chave no mercado brasileiro, segundo mercado de alimentos para animais de estimação após os Estados Unidos em nível mundial. A empresa $\mathrm{D}$ tem posições-chave em gado leiteiro, alimentação de cavalos e aquicultura. Com a aquisição da empresa D, a D1 confirmou a sua estratégia global de alimentos para animais de estimação o que permite que ela passe a ocupar uma posição-chave no mercado brasileiro. 


\subsection{Proximidade do core business (atividade central) e impactos da aquisição para as empresas - matriz/adquirente e subsidiária/adquirida}

A revisão de literatura levantou a importância do parentesco de negócios para o sucesso do relacionamento entre matriz e subsidiária. Tang e Rowe (2012) observaram que o parentesco de negócios, definido como a medida com que uma subsidiária está relacionada com a atividade principal (core business) de sua matriz, tem um efeito positivo sobre o desempenho da subsidiária.

Buscando avaliar o tema da proximidade do core business entre matriz e subsidiária, foi possível observar, de acordo com os dados analisados que no caso específico da empresa A, a aquisição da empresa pela multinacional francesa A1 foi muito positiva e de muito aprendizado para os dois lados. Antes da parceria, a multinacional francesa ainda não possuía uma empresa de vídeo segurança, trabalhando apenas com controle de acesso e motores para abrir e fechar portões e janelas. Então a empresa brasileira transmitiu o conhecimento à matriz francesa sobre vídeo segurança, tecnologia e mercado, envolvendo os conhecimentos sobre os clientes, fornecedores e toda a cadeia logística, o que resultou na criação, na matriz francesa, de um departamento na área de vídeo segurança.

Para a empresa A (brasileira), a matriz transferiu conhecimentos que ajudaram a resolver problemas relacionados à qualidade dos produtos. A A1 utilizou o mecanismo de treinamentos e mandou uma equipe para o Brasil para ensinar o pessoal da A sobre o desenvolvimento de produtos de qualidade. Além disso, os brasileiros também se beneficiaram do ponto de vista tecnológico e mercadológico com o aprendizado sobre a integração de produtos, na área de automação residencial - uma área que integrava todas as tecnologias.

No segundo caso analisado, da empresa B, na época da aquisição, a adquirente B1 não tinha presença nas Américas, então viu a B como uma oportunidade de entrar no continente. Além disso, a B1 também percebeu na brasileira B uma nova oportunidade, pois tinha um escopo e um foco de produtos um pouco diferente. A multinacional B1 sempre focou em grandes empresas e desenvolveu tecnologia para grandes corporações, já a B sempre desenvolveu soluções mais focadas para pequenos e médios negócios.

Com relação aos benefícios que a matriz estrangeira trouxe para a $\mathrm{B}$, ela não só incentivou a geração de inovação, como até mesmo colaborou para um processo de expansão do produto da brasileira para pequenos e médios lojistas. Nesse contexto, hoje a empresa 
brasileira investe muito mais em tecnologia do que investia no passado e inclusive trabalha com a ideia de fazer a expansão de seu produto para outros países do mundo.

No caso da empresa C que foi adquirida pelo Grupo C1 em 2016 e se tornou C/C1, a análise dos dados mostrou que as duas empresas, mas principalmente a matriz, foram beneficiadas. A multinacional, Grupo $\mathrm{C} 1$, sempre foi muito focada em alimentação e refeição e o que chamou a atenção do Grupo para a empresa $\mathrm{C}$ foi a diversidade e a visão de futuro da brasileira $\mathrm{C}$ que trabalha dentro de cinco indústrias: é emissora de cartões, é uma bandeira, assim como Visa e MasterCard, trabalha no setor de pagamentos eletrônicos com rede própria, é processadora de meios eletrônicos de pagamentos e também é administradora de cartão de crédito.

Um dos maiores benefícios da compra da empresa C pelo Grupo multinacional C1 para a empresa brasileira está no crescimento da empresa $\mathrm{C}$, no aumento do número de clientes e na valorização e respeito que a $\mathrm{C}$ passou a receber do mercado.

No quarto caso estudado, o core business das duas empresas D e D1 é nutrição animal. As duas empresas são muito bem alinhadas. O primeiro benefício que pôde ser observado com a compra da empresa $D$ para a multinacional $D 1$ foi a fatia de mercado $\mathrm{e} o$ conhecimento/knowhow que a D possuía no segmento Pet no mercado nacional e internacional. O segundo benefício para a multinacional foi que a $\mathrm{D}$ sempre atuou muito fortemente em animais de produção, principalmente gado leiteiro. Então, as inovações e o conhecimento nessa linha de ruminantes, principalmente gado leiteiro, foram relevantes para a multinacional D1.

Com relação à empresa $\mathrm{D}$, ela foi beneficiada, do ponto de vista da geração de inovação, modernização, melhoria de fórmula e também melhoria de processo fabril. De acordo com os dados analisados, é possível observar que atualmente a empresa $\mathrm{D}$ tem um produto diferenciado e uma embalagem mais tecnificada. Nesse contexto, foi possível perceber que a empresa com os investimentos recebidos da matriz em pessoal e em capital resultaram no desenvolvimento de inovações, como por exemplo, inovações incrementais - mesmos produtos, com adaptações nas embalagens, entre outras. 


\subsection{Proximidade cultural}

A literatura consultada que aborda as relações entre a matriz e as subsidiárias de empresas multinacionais e a geração de inovação nas subsidiárias levantou temas como os desafios de se gerenciar a transferência de conhecimentos entre a matriz e subsidiárias, principalmente se essas se localizam em contextos geográficos e culturais distantes (Lucas, 2006; Qin et al., 2008; Boh et al., 2013). Nesse contexto, pesquisadores explicam que a transferência de tecnologia entre matriz e subsidiárias pode ser mais complexa quando as partes envolvidas no processo possuem diferenças culturais (Sunaoshiet al., 2005).

Com relação à questão das diferenças culturais, a interpretação dos dados mostrou que no caso da empresa $\mathrm{A}$, as empresas eram próximas culturalmente, principalmente com relação a questões como o entendimento da meritocracia, o desenvolvimento das pessoas e a liderança nas empresas. A proximidade também pôde ser percebida com relação aos aspectos regionais da A e da A1. A multinacional A1 ficava em uma região da França chamada Cluses. Cluses é uma espécie de Vale do Silício dos motores. É uma cidadezinha com 30.000 habitantes, no interior da França, ao lado das montanhas e focada em tecnologia. Essa foi vista como uma grande semelhança entre as duas empresas, pois a empresa A está situada entre montanhas, na cidade de Santa Rita do Sapucaí, uma cidade com 40.000 habitantes, que também é focada tecnologia. Além disso, a língua não foi entrave, as empresas se comunicavam em inglês.

No caso do relacionamento entre a empresa B e a multinacional B1, de acordo com os entrevistados, não houve problemas. A B1 mantém um distanciamento com relação à forma como os gestores da B administram a empresa. Apesar de ser uma empresa alemã, a B1 é uma empresa global, presente no mundo todo e a língua oficial na matriz em Munique é o inglês. Nesse contexto, por ser um time multicultural, a matriz não representou nenhum problema cultural para a empresa brasileira.

No caso específico da $\mathrm{C} / \mathrm{C} 1$, o gestor explicou a empresa precisou lidar não somente com a diferença cultural de duas empresas - matriz e subsidiária - mas com diferenças culturais de 4 empresas e que esse foi um dos maiores desafios que precisaram enfrentar. Quando a $\mathrm{C}$ foi adquirida pelo Grupo C1 a empresa francesa também havia adquirido outras duas empresas brasileiras e então foi precisar trabalhar para a integração das três empresas brasileiras e da francesa. Foi preciso que todos entendessem sobre os negócios das empresas diferentes e 
ajustassem e unissem essas empresas. Além disso, também ficaram claras as diferenças entre a cultura brasileira e a francesa e as diferenças das línguas.

No caso da $\mathrm{D}$, os dados analisados mostraram que houve até certo ponto um choque cultural entre as duas empresas, principalmente devido às diferenças com relação à forma de trabalho e à tecnologia. Segundo o gestor entrevistado, a aquisição da D representou uma mudança radical para a empresa adquirida, pois a empresa não possuía os softwares e as tecnologias de informação com que as outras empresas do grupo D1 estavam acostumadas a trabalhar. As técnicas de produção também sofreram mudanças.

\subsection{Autonomia diante da matriz}

De acordo com a literatura revisada, maiores graus de autonomia para a subsidiária e descentralização na rede da multinacional podem permitir à subsidiária assumir um papel mais ativo na prospecção de novos conhecimentos e mercados e, consequentemente, na geração de inovação (Ferreiraet al., 2013). As entrevistas analisadas mostraram que após a aquisição da empresa $\mathrm{A}$, essa empresa teve muita autonomia com relação à matriz para desenvolver seu trabalho. De acordo com as análises, como os franceses da empresa multinacional A1 não eram do ramo de vídeo segurança, eles mantiveram um CEO no Brasil, um controlador de finanças e também investiram em apresentações e treinamentos sobre a estratégia da A1, mas vale ressaltar que o contrato entre as empresas estabelecia que durante um período de cinco anos, a A1 não seria dona integral da empresa, mantendo os empreendedores iniciais como donos em conjunto com a A1.

No caso da B, após a aquisição, a alemã B1 manteve um distanciamento para garantir à B autonomia para administrar a empresa e não enviou um gestor alemão para a empresa no Brasil.

Especificamente no caso da C/C1, após a integração das empresas ao Grupo C1 francês, apesar de muita autonomia, algumas questões como o padrão nacional ou mundial de imagem, precisoude ajustes. No caso dos produtos, a parceria funciona mais ou menos como em uma sociedade, 'enquanto a subsidiária brasileira estiver dando lucro, os brasileiros têm autonomia'.

Com relação à questão da autonomia dentro da $\mathrm{D}$ para desenvolver seu trabalho, de acordo com os dados analisados, a empresa trabalha no marketing com vários gerentes de 
produtos que são gerentes 'espécie específicos', ou seja, há um gerente de produto Pet, um gerente de produto equinos, um gerente de produto ruminantes, corporativos, que decidem e atingem todas as marcas. Esses gerentes são espécie específicos da D1 do Brasil. Então esse gestor é o responsável por toda a comunicação, lançamento de produto, mudanças de produtos. Nesse caso específico da D1, a matriz tem as decisões mais centralizadas, e todo o processo decisório tem que passar pela D1.

\subsection{Motivação e incentivos recebidos da matriz para a geração de inovação}

De acordo com a literatura consultada, o relacionamento entre a matriz e a subsidiária pode contribuir positivamente para a inovação na subsidiária (Keuppet al., 2011). Os autores sugeriram que a matriz pode melhorar ativamente o desempenho de uma subsidiária no contexto da geração de inovação.

Procurando entender o papel da matriz e questões como a motivação e os incentivos da matriz para a geração de inovação, foi possível observar com a análise dos dados que no caso da A, a empresa foi incentivada e recebeu investimentos para trabalhar com a integração de produtos. A empresa estava acostumada a vender os produtos isoladamente e passou a ter uma preocupação muito maior em fazer com que todos esses produtos se comunicassem. A matriz foi responsável por ensinar a A sobre essa questão.

No caso da empresa B, a multinacional B1 viu a empresa não só como uma oportunidade de entrar no continente sul-americano, mas de trabalhar com um escopo de produtos um pouco diferente do que ela possuía. A B1 tem tecnologia financeira para grandes corporações no mundo todo e a empresa B era diferente, porque tinha uma solução mais focada em pequenos e médios negócios, um produto complementar ao da B1. Em razão disso, a matriz tem incentivado o desenvolvimento de novos produtos e inovação como também a expansão do produto que a B tem para pequenos e médios lojistas. De acordo com os dados analisados, hoje a B investe muito mais em tecnologia do que investia no passado e trabalha com a possibilidade de expansão de seu produto para outros países do mundo.

Com respeito ao caso da empresa $\mathrm{C}$, desde a aquisição, a matriz ainda não tem exatamente promovido a criação de produtos totalmente novos, porque além do trabalho de integração da empresa brasileira, formada a partir de três empresas, está procurando entender o mercado brasileiro e a diversidade de produtos com os quais trabalha a empresa $\mathrm{C}$. 
No caso específico da empresa $\mathrm{D}$, a matriz incentivou a geração de inovação e a criação de novos produtos. Um dos principais segmentos novos trabalhados pela empresa D é para o produto 'aqua' que é o produto para peixes. E até então, a D não tinha produtos com tecnologia, principalmente, em rações iniciais para peixe. Esse foi um dos principais benefícios que a empresa teve ao fazer parte do Grupo D1. Essa pode ser vista como uma inovação radical, um produto totalmente novo para a empresa brasileira.

\section{Considerações finais}

Partindo das literaturas de negócios internacionais, transferência de conhecimento e gestão da inovação que colaboram para o entendimento da inovação em empresas multinacionais, o presente estudo além de propor a elaboração de um quadro teórico para ilustrar fatores que permeiam o relacionamento entre matriz e subsidiária e apontar a influência desses fatores na geração de inovação na subsidiária, também analisou a realidade por meio de um estudo de casos múltiplos (envolvendo quatro casos), visando entender a seguinte questão: como o relacionamento entre matriz e subsidiária e como a atuação da própria matriz interferem nos processos de inovação na subsidiária.

A revisão de literatura levantou a importância da proximidade do (core business) entre matriz e subsidiárias para o sucesso do relacionamento e consequentemente para o desempenho positivo da subsidiária na geração de inovação. No estudo de casos múltiplos desenvolvido, todas as subsidiárias pesquisadas não possuíam exatamente a mesma atividade principal de suas matrizes, o que não representou em nenhum dos casos um aspecto negativo para o relacionamento entre matriz e subsidiária e para a geração de conhecimentos novos. Na realidade dos casos observados, as diferenças com relação a esse ponto analisado colaboraram para o aprendizado dos dois lados. Nos casos da empresa B e da empresa D, as diferenças com relação ao core business entre matriz e subsidiária colaboraram para o começo do desenvolvimento de novos produtos ou serviços nas subsidiárias - o que nesses dois casos representou produtos totalmente novos para essas empresas. Essa diferença também colaborou para o acesso a informações sobre a geração de uma linha de produtos integrados e com qualidade superior no caso da empresa A - representando uma inovação na forma de trabalhar (inovação organizacional); e para a criação de uma nova proposta de trabalho ou plataforma 
que agregasse vários serviços no caso da $\mathrm{C}$ - nova forma de estruturar os serviços da empresa (inovação de processo para a empresa).

A literatura consultada também chamou a atenção para dificuldades enfrentadas por subsidiárias de empresas multinacionais para se relacionar com a matriz que podem ser causadas pela distância cultural, podendo prejudicar a inovação. Analisando esse aspecto, os casos estudados mostraram que as empresas A e B não tiveram problemas com relação a esse aspecto e que a proximidade cultural entre essas empresas e suas matrizes colaborou para facilidade das relações. No caso da empresa C, as diferenças culturais e organizacionais surgiram não só a partir do relacionamento novo com a matriz, mas também do relacionamento com outras duas empresas que passaram a fazer parte do grupo. Nesse caso, essas diferenças precisaram ser resolvidas para que o negócio pudesse acontecer e fez nascer uma empresa completamente nova, mais flexível e inovadora. No caso da empresa D, a distância cultural se mostrou presente e mais relacionada à diferença entre as culturas empresariais tecnológicas, o que se traduziu em benefícios de infraestrutura e aprendizado para a subsidiária. Com a instalação de softwares e redes internas, a empresa D mudou a sua forma de trabalhar (inovação organizacional).

O presente estudo também direcionou atenção à questão da autonomia, tendo em vista que de acordo com a literatura, graus mais elevados de autonomia da subsidiária perante a matriz podem permitir um papel mais ativo na prospecção de novos conhecimentos e mercados e, consequentemente, na geração de inovação na subsidiária. Os casos pesquisados mostraram que três empresas possuíam bastante autonomia para desenvolver suas atividades: A, B e C. Já a empresa $\mathrm{D}$ teve sua estrutura organizacional modificada pela matriz, justamente para permitir um maior controle sobre a subsidiária.

Por fim, procurando entender o papel da matriz e questões como os investimentos e a motivação recebidos da matriz para a geração de inovação na subsidiária, foi possível observar com os casos que todas as empresas perceberam benefícios e foram incentivadas a gerar produtos e serviços novos ou a realizar algum tipo específico de inovação. A empresa A foi incentivada e recebeu investimentos e treinamento para trabalhar com a integração de produtos e com qualidade superior. A empresa B recebeu incentivos e investimentos para a área de segurança e para desenvolver uma expansão de sua linha de produtos. A empresa C inovou de forma organizacional e está construindo uma plataforma para integrar os serviços dentro do Grupo. A empresa D tem recebido investimentos e treinamento para se modernizar e melhorar 
do ponto de vista tecnológico o seu portfólio com inovações incrementais, e passou a lançar novos produtos, especificamente, novos para a empresa.

Diante dos casos analisados foi possível perceber que em sua maioria os processos de aquisição que incorporaram as empresas pesquisadas na rede das multinacionais adquirentes representaram benefícios para os dois lados: subsidiária e matriz, principalmente do ponto de vista da aprendizagem e da geração de conhecimentos novos dentro das matrizes e das subsidiárias. Os casos pesquisados mostraram que todas as subsidiárias tiveram após os processos de incorporação pela multinacional algum tipo de inovação desencadeado.

\section{Agradecimentos}

Os autores agradecem à Fundação de Amparo à Pesquisa de Minas Gerais (FAPEMIG) pelo apoio financeiro.

\section{Referências bibliográficas}

Achcaoucaou, F., Miravitlles, P., \& León-Darder, F. (2016). Do we really know the predictors of competence-creating R\&D subsidiaries? Uncovering the mediation of dual network embeddedness. Technological Forecasting \& Social Change, 116, 181-195.

Almeida, P., \& Phene, A. (2004). Subsidiaries and knowledge creation: the influence of the MNC and host country on innovation. Strategic Management Journal, 25, 847-864.

Andrade, E. F. S., Oliveira, J., Maccari, E. A., \& Hollnagel, H. (mai./ago. 2018). Inovação em um contexto isomórfico nos Programas De Pós-Graduação Stricto Sensu em administração. Revista Gestão \& Tecnologia, 18(2), 100-127.

Andreassi, T. (2007). Gestão da inovação tecnológica. São Paulo: Thomson Learning.

Baglieri, E., Bruno, M., Vasconcellos, E., \& Grando, A. (2014). Innovation process in the automobile MNCs: implications for the role of the international subsidiaries. Int. J. Automotive Technology and Management, 14(1), 82-98.

Beddi, H., \& Mayrhofer, U. (2013) Headquarters-subsidiaries relationships of French multinationals in emerging markets.The Multinational Business Review, 21(2), 174-194.

Birkinshaw, J., Hood, N., \& Josnsson, S. (1998). Building firm-specific advantages in multinational corporations: the role of subsidiary initiative. Strategic Management Journal, 19(3), 221- 241.

Boehe, D. M. (Jan./Mar. 2007). Desenvolvimento de produtos em subsidiárias de empresas multinacionais no Brasil. RAE Revista de Administração de Empresas, 47(1), 33-45. 
Boehe, D. M. (Jan./Mar. 2010). The influence of coordination mechanisms on new product development in MNC subsidiaries. BAR, Curitiba, 7(1), 79-97.

Boh, W. F. Nguyen, T. T., \& Xu, Y. (2013). Knowledge transfer across dissimilar cultures. Journal of Knowledge Management, 17(1), 29-46.

Cabral, J. E. O. (Oct./Dec. 2007). Determinantes da propensão para inovar e da intensidade inovativa em empresas da indústria de alimentos do Brasil. RAC. Curitiba, 11(4), 87-108.

Cantwell, J. (2016). Innovation and international business. Industry and InnovatIon, 24(1), $41-60$.

Catwell, J. A., \& Mudambi, R. (December, 2005).MNE competence-creating subsidiary mandates. Strategic Management Journal, 26(12), 1109-1128.

Christensen, J. F. (1995). Asset profiles for technological innovation. Research Policy, 24(5), 727-745.

Christensen, C. M. (1997). The Innovators dilemma. Boston/USA: Harvard Business School Press.

Ciabuschi, F.; Dellestrand, H., \& Martín, O. M. (November, 2011). Internal embeddedness, headquarters involvement, and innovation importance in multinational enterprises. Journal of Management Studies,48(7), 1612-1639.

Ciabuschi, F.; Forsgren, M.; \& Martín, O. M. (2017). Value creation at the subsidiary level: testing the MNC headquarters parenting advantage logic. Long Range Planning, xxx, 115.

Cohen, W. M., \& Levinthal, D. A. (1990). Absorptive capacity: a new perspective on learning and innovation. Administrative Science Quarterly, 1(35), 128-152.

Corley, K. G., Gioia, D. A. (Jun. 2004). Identity ambiguity and change in the wake of a corporate spin-off. Administrative Science Quaterly, 49(2), 173-208.

Costa, S., Borini, F. M., Amatucci, M. (Aug. 2013). Inovação global de subsidiárias estrangeiras localizadas em mercados emergentes. Revista de Administração Contemporânea, Curitiba, 17(4), 459-478.

Du, J., \& Williams, C. (2017). Innovative projects between MNE subsidiaries and local partners in China: exploring locations and inter-organizational trust. Journal of International Management, 23(1), 16-31.

Ferreira, M. P., Beltrão, J. S., \& Almeida, M. R. (2013). Relações de controle e autonomia entre multinacionais e subsidiárias: um estudo de caso de multinacional norte-americana em Portugal. Internext: Revista eletrônica de negócios internacionais, São Paulo, 8(3), 2037.

Figueira, M., Luchesi, P. H. M., Silva, G. F., \& Calegario, C. L. L. (2017). Empresas multinacionais e a influência da atuação da matriz na intensidade inovativa da subsidiária. In XVII Congreso Latino-iberoamericano de Gestión Tecnológica (ALTEC 2017), Ciudade de México, México. 
Figueiredo, P. N. (2011). The role of dual embeddedness in the innovative performance of MNE subsidiaries: Evidence from Brazil. Journal of Management Studies, 48(2), 417-440.

Gavira, M. O. (2008). Gestão da inovação em subsidiárias de multinacionais do setor eletroeletrônico instaladas no Brasil. Tese (Doutorado), Universidade Estadual de Campinas, Campinas, S.P.

Ho, Y. (2014). Multilateral knowledge transfer and multiple embeddedness. The Multinational Business Review, 22(2), 155-175.

Kawai, N., \& Strange, R. (2014). Subsidiary autonomy and performance in Japanese multinationals in Europe. International Business Studies, 23(3), 504-515.

Keupp, M. M., Palmié, M., \& Gassmann, O. (2011). Achieving subsidiary integration in international innovation by managerial "tools". Management International Review, 51, 213-239.

Lucas, L. M. (2006). The role of culture on knowledge transfer: the case of the multinational corporation. The Learning Organization, 13(3), 257-275.

Machado, M. A., Bauer, E. A. (abr-jun/2014). O papel das subsidiárias em países emergentes na geração de vantagem competitiva para multinacionais estrangeiras: o caso da subsidiária brasileria da ClarksInternational. GEPROS. Gestão da Produção, Operações e Sistemas, Bauru, 9(2), 65-82.

Minayo, M. C. S (2000). O desafio do conhecimento: pesquisa qualitativa em saúde, São Paulo: Hucitec.

Minbaeva, D., Pedersen, T, Bjorkman,I., Fey, C. F., \& Park, H. J. (2003). MNC knowledge transfer, subsidiary absorptive capacity and HRM. Journal of International Business Studies, 34, 586-599.

Moreira, D. A., \& Queiroz, A. C. S. (2007). Inovação organizacional e tecnológica. São Paulo: Thomson Learning.

Mudambi, R., Piscitello, L., \& Rabbiosi, L. (2014). Reverse knowledge transfer in MNEs: subsidiary innovativeness and entry modes. Long Range Planning, 47, (1,2). 49-63.

Najafi-Tavani, Z., Zaefarian, G., Naudé, P., \& Giroud, A. (2015). Reverse knowledge transfer and subsidiary power. Industrial Marketing Management, 48, 103-110.

Nemoto, M. C. M. O., Santos, G. Z. V.; \& Pinochet, L. H. C. (jan./abr. 2018). Adoção de inovação: internet das coisas para melhoria de desempenho de sustentabilidade na Klabin. Revista Gestão \& Tecnologia, 18(1), 197-224.

OECD/Eurostat. (2005). OECD Proposed Guidelines for Collecting and Interpreting Technological Innovation Data - Oslo Manual. Paris: OECD.

Piscitello, L., \& Rabbiosi, L. (2006). How does knowledge transfer from foreign subsidiaries affect parent companies' innovative capacity? DRUID Working Papers, (06-22).

Qin, C., Ramburuth, P., \& Wang, Y. (2008) Cultural distance and subsidiary roles in knowledge transfer in MNCs in China. Chinese Management Studies, 2(4), 260-280.

Schleimer, S. C., \& Pedersen, T. (2013). The effects of MNC parent effort and social structure on subsidiary absorptive capacity. Journal of International Business Studies, 45, 303-320.

$@$ Q Revista Gestão \& Tecnologia, Pedro Leopoldo, v. 19, n. 5, p. 143-166, out./dez. 2019 
Schreiber D., Vilela, D. C. J., Vargas, L. M., \& Maçada A. C. G. (2011). Knowledge transfer in product development: an analysis of Brazilian subsidiaries of multinational corporations. Brazilian Administration Review, 8(3), 288-304.

Scott, P. S., \& Gibbons, P. T. (2009). How subsidiaries are battling to survive and grow. Strategy and Leadership, 37(4), 43-47.

Silva, E. D., Gaspar, M. A., Costa, I., \& Magalhães, F. L. F. (jan./abr. 2018). Gestão do conhecimento de service desk: estudo de caso da integração em empresas adquiridas por uma corporação multinegócios. Revista Gestão \& Tecnologia, 18(1), 140-170.

Silveira, F. F.; Sbragia, R.; Lopez-Vega, H.; \& Tell, F. (2017). Determinants of reverse knowledge transfer for emerging market multinationals: the role of complexity, autonomy and embeddedness. Revista de Administração, (52), 176-188.

Strauss, A and J Corbin (1990). Basics of qualitative research: grounded theory procedures and techniques, London: Sage Publications.

Sunaoshi, Y., Kotabe, M., \& Murray, J. Y. (2005). How technology transfer really occurs on the factory floor: a case of a major Japanese automotive die manufacturer in the United States. Journal of World Business,40, 57-70.

Tang, J., \& Rowe, W. G. (2012). The liability of closeness: business relatedness and foreign subsidiary performance. Journal of World Business, 47, 288-296.

Tsai, C., \& Wen, C. (2009). The effects of relational embeddedness on entrepreneurship Taiwanese subsidiaries in China. International Journal of Entrepreneurship Behaviour, 15 (5), 453-472.

Yin, R. K. (2010). Estudo de caso: planejamento e métodos. Porto Alegre: Bookman.

Zander, U., \& Kogut, B. (1995). Knowledge and speed of transfer and imitation of organizational capabilities: an empirical test. Organizatin Science, 6(1), 76-92. 